\title{
A detailed study of the hysteresis in La0.67Ca0.33MnO3
}

\author{
Neves Bez, Henrique; Nielsen, Kaspar Kirstein; Smith, Anders; Bahl, Christian R. H.
}

\section{Published in:}

Journal of Magnetism and Magnetic Materials

Link to article, DOI:

10.1016/j.jmmm.2016.05.011

Publication date:

2016

Document Version

Peer reviewed version

Link back to DTU Orbit

Citation $(A P A)$ :

Neves Bez, H., Nielsen, K. K., Smith, A., \& Bahl, C. R. H. (2016). A detailed study of the hysteresis in $\mathrm{La}_{\mathrm{C}} \mathrm{Ca}_{33} \mathrm{MnO}_{3}$. Journal of Magnetism and Magnetic Materials, 416, 429-433.

httBs.7/doi.8133/10.1016/j.jmmm.2016.05.011

\section{General rights}

Copyright and moral rights for the publications made accessible in the public portal are retained by the authors and/or other copyright owners and it is a condition of accessing publications that users recognise and abide by the legal requirements associated with these rights.

- Users may download and print one copy of any publication from the public portal for the purpose of private study or research.

- You may not further distribute the material or use it for any profit-making activity or commercial gain

- You may freely distribute the URL identifying the publication in the public portal

If you believe that this document breaches copyright please contact us providing details, and we will remove access to the work immediately and investigate your claim. 


\title{
A detailed study of the hysteresis in $\mathrm{La}_{0.67} \mathrm{Ca}_{0.33} \mathrm{MnO}_{3}$
}

\author{
Henrique N. Bez ${ }^{\mathrm{a}, *}$, Kaspar K. Nielsen ${ }^{\mathrm{a}}$, Anders Smitha ${ }^{\mathrm{a}}$, Christian R. H. Bahl ${ }^{\mathrm{a}}$ \\ ${ }^{a}$ Department for Energy Conversion and Storage, Technical University of Denmark, Risø Campus, Frederiksborgvej 399, \\ DK-4000 Roskilde, Denmark
}

\begin{abstract}
We report a thorough study of the thermal hysteretic behaviour of a single phase sample of the magnetocaloric material $\mathrm{La}_{0.67} \mathrm{Ca}_{0.33} \mathrm{MnO}_{3}$. Previous reports in the literature have variously found hysteretic and non-hysteretic behaviour. We show the importance of measuring under carefully defined heating and cooling procedures. Careful analysis of the specific heat, measured at five different temperature ramp rates, and the magnetic entropy change indicates that there is no observable hysteresis, even though the behaviour of both quantities is consistent with a first-order phase transition. We discuss the reasons for this and for the differing results previously found.
\end{abstract}

Keywords: Magnetocaloric effect, Hysteresis, Phase transition, Manganites

\section{Introduction}

A broad range of materials display the magnetocaloric effect. An interesting example is the Lamanganites, in particular $\mathrm{La}_{0.67} \mathrm{Ca}_{0.33} \mathrm{MnO}_{3}$ which 5 has been investigated for several years as a possible material for magnetocaloric applications [1, 2, 3. Of particular interest is whether the phase transition is of first order or of second order. As a general rule, materials with a first-order phase transition (FOPT) can exhibit a larger magnetocaloric effect than materials with a second-order phase transition (SOPT). On the other hand thermal hysteresis, which gives rise to losses when the materials are applied in thermodynamic cycles, is associated with FOPT. This makes it of both theoretical and practical importance to investigate the hysteresis of magnetocaloric materials.

$\mathrm{La}_{0.67} \mathrm{Ca}_{0.33} \mathrm{MnO}_{3}$ has been the subject of much research over the past decades due to its many interesting properties. Even so, it remains a much debated question whether the material undergoes a FOPT or a SOPT [4, 5]. Furthermore, thermal hysteresis has been studied in this material with contradicting results [1, 5, 6, 7]. One complication 25 is that different processing routes may lead to dif-

\footnotetext{
* Corresponding author

Email address: hnbe@dtu.dk (Henrique N. Bez)
}

ferent properties [8], e.g. the reported Curie temperatures vary between 250 to $270 \mathrm{~K}$. Furthermore, care has to be taken when investigating thermal hysteresis in FOPT to avoid sampling metastable mixed states in the transition region between the paramagnetic and ferromagnetic states. It has previously been shown that for observation of the true thermal hysteresis it is necessary to 'reset' the sample between measurements by cooling or heating 35 to a temperature where only one phase is present [9, 10.

One of the models used to shed some light on the magnetic phase transition of materials is the Bean-Rodbell model [11. Bean and Rodbell have 40 proposed that the exchange constant, and therefore $T_{\mathrm{C}}$, varies linearly with the lattice spacing as $T_{\mathrm{C}}=T_{0}\left[1+\beta \frac{V-V_{0}}{V_{0}}\right]$, where $V$ is the unit cell volume, $\mathrm{T}$ is temperature, and $T_{0}$ and $V_{0}$ are temperature and unit cell volume in the absence of ex45 change interaction, respectively. The parameter denoted by $\beta$ controls the strength of the spin-lattice coupling. Moreover, one of the implications of this model is the introduction of possible irreversibilities, such as hysteresis. One of the parameters so of the model, $\eta$, can control whether the transition is first order, $\eta>1$, or second order, $\eta \leq 1$. This model has been used with good agreement with measured properties of some compositions of $\mathrm{La}_{1-x} \mathrm{Ca}_{x} \mathrm{MnO}_{3}$, where it was observed $\eta$ values 
above 1 12, i.e. first order phase transition. Furthermore, literature has shown that by superimposing a Gaussian distribution in the Curie temperature, one can simulate the effect of chemical inhomogeneities on the properties 13 .

Here we use measurements of $c_{p}$ and both indirect and direct measurements of the isothermal entropy change $\Delta s$ to evaluate the phase transition of $\mathrm{La}_{0.67} \mathrm{Ca}_{0.33} \mathrm{MnO}_{3}$. The indirect measurement is done by measuring the magnetization as a function of field and temperature and subsequently using a numerical integration of the Maxwell relation $\Delta s=\mu_{0} \int(\partial M / \partial T) d H$, while the direct measurement uses calorimetry [14, 15, 16]. The latter avoids the approximations arising from the nu-

70 merical integration and provides a very stable temperature for the measurements. Below we report studies of the thermal hysteretic and FOPT behaviour by means of measurements of $\Delta s$ and $c_{p}$, and by the implementation of Bean-Rodbell model

${ }_{75}$ of $\mathrm{La}_{0.67} \mathrm{Ca}_{0.33} \mathrm{MnO}_{3}$ produced by solid-state reaction.

\section{Experimental Procedure}

$\mathrm{La}_{0.67} \mathrm{Ca}_{0.33} \mathrm{MnO}_{3}$ polycrystalline material was synthesized by solid-state reaction. $\mathrm{La}_{2} \mathrm{O}_{3}, \mathrm{CaCO}_{3}{ }_{110}$ and $\mathrm{MnO}_{2}$ in powder form were ground and mixed stoichiometrically by roll milling during $48 \mathrm{~h}$ with a rotation speed of $180 \mathrm{rpm}$. The powder was then calcinated at $1123 \mathrm{~K}$ for $24 \mathrm{~h}$. Subsequently, the powder was isostatically pressed into pellets ${ }^{115}$ 85 and sintered at $1403 \mathrm{~K}$ for $48 \mathrm{~h}$. Both calcination and sintering were performed in air. X-ray diffraction (XRD) measurements were performed in a Brucker D8 X-ray diffractometer at ambient temperature and pressure, under $\mathrm{Cu}$ radiation. The ${ }^{120}$ 90 specific heat, $c_{p}$, was measured at different temperature rates in a custom-built differential scanning calorimeter (DSC) [17] under several applied fields. The isothermal entropy change was measured for every $0.25 \mathrm{~K}$ in the same device, by keeping the ${ }^{125}$

95 temperature fixed and varying the applied magnetic field. We used four different measurement protocols around the transition temperature to avoid the appearance of mixed states when characterizing the entropy change:

i. From the ferromagnetic state (FM) towards 130 paramagnetic state $(\mathrm{PM})$ with a reset temperature at $240 \mathrm{~K}$ and going from $0 \mathrm{~T}$ to $1.0 \mathrm{~T}$;

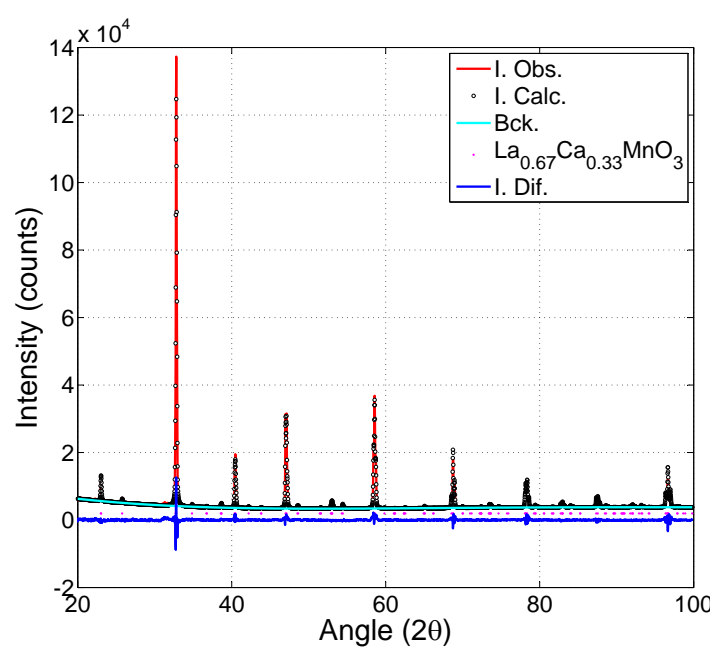

Figure 1: XRD pattern and Rietveld refinement of the perovskite showing the desired single phase structure.

ii. From FM towards PM with a reset temperature at $240 \mathrm{~K}$ and going from $1.0 \mathrm{~T}$ to $0 \mathrm{~T}$;

iii. From PM towards FM with a reset temperature at $290 \mathrm{~K}$ and going from $0 \mathrm{~T}$ to $1.0 \mathrm{~T}$;

iv. From PM towards FM with a reset temperature at $290 \mathrm{~K}$ and going from $1.0 \mathrm{~T}$ to $0 \mathrm{~T}$;

When resetting, the sample is taken to the chosen reset temperature and allowed to thermally equilibrate. Low temperature magnetisation was measured in a high field VSM (Cryogen Free Measurement System - $16 \mathrm{~T}$ ) at $10 \mathrm{~K}$ from 0 to $10 \mathrm{~T}$, with a rate of $0.1 \mathrm{~T} / \mathrm{min}$ to find the saturation magnetisation. Moreover, a LakeShore VSM, model 7407, was used to measure isothermal magnetisation around the transition temperature in order to calculate $\Delta s$, and compare with the direct measurements in the calorimeter. These $\Delta s$ measurements were done from $0 \mathrm{~T}$ to $1.6 \mathrm{~T}$ and for every $0.5 \mathrm{~K}$. The demagnetization effects were taken into account when processing the data, assuming a prism shaped sample 18. The sample has the approximate dimensions $3.8 \times 1.2 \times 1.2 \mathrm{~mm}^{3}$. The field orientation in the isothermal magnetization measurements was along the long axis to minimize demagnetization, while in the DSC the field was along the short axis, due to size incompatibility.

\section{Results}

LCMO has an O-type orthorhombic structure, with a distortion of the $\mathrm{MnO}_{6}$ octahedra due to the Jahn-Teller effect 19. Figure 1 shows the XRD 


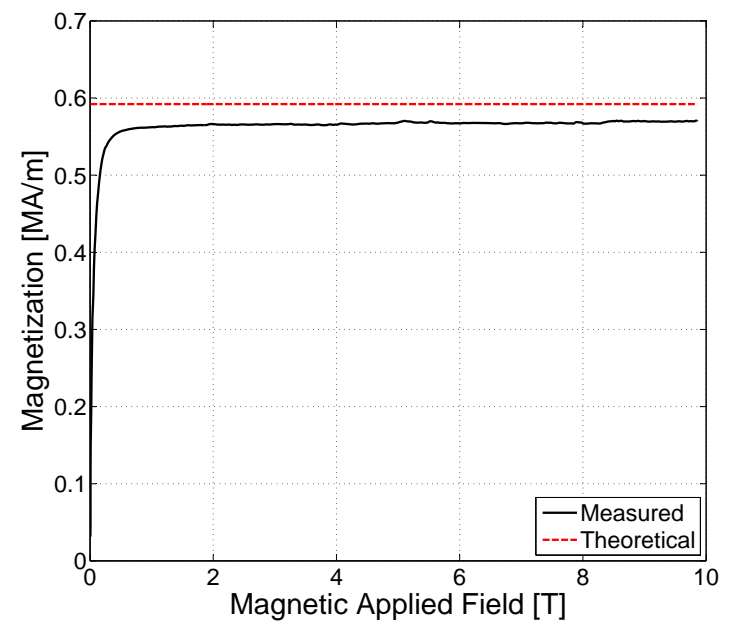

Figure 2: Magnetisation measurement at $10 \mathrm{~K}$, from $0 \mathrm{~T}$ to $10 \mathrm{~T}$ at a rate of $0.1 \mathrm{~T} / \mathrm{min}$. The solid line represents the measurement, and the dashed red line is the theoretical value of the saturation magnetisation.

pattern and Rietveld refinement of the polycrystalline $\mathrm{La}_{0.33} \mathrm{Ca}_{0.67} \mathrm{MnO}_{3}$. From the Rietveld re- ${ }_{16}$ of $\mathrm{La}_{0.33} \mathrm{Ca}_{0.67} \mathrm{MnO}_{3}$ where the unit cell parameters $a, b$ and $c$ were calculated to be $5.47154(7) \AA$, $5.45690(1) \AA$ and $7.7086(1) \AA$, respectively, resulting in a unit cell with a volume of $230.162(7) \AA^{3}$, 170 wich is in agreement with the literature 4 . Furthermore, Figure 2 shows the magnetisation measurement at $10 \mathrm{~K}$, where the solid line is the measurement and the dashed red line is the theoretical saturation value calculated as follows:

$$
M_{s}=g \mu_{\mathrm{B}} J \rho_{s} .
$$

Here, $g$ is the Landé factor, $\mu_{B}$ is the Bohr magneton, $J$ is the total quantum angular momentum and $\rho_{s}$ is the magnetic spin density. The Landé factor ${ }^{180}$ used is $2, \mathrm{~J}$ is 1.835 (average on the ratio of $\mathrm{Mn}^{+3}$ and $\mathrm{Mn}^{+4}$ ) and the spin density is calculated simply by the number of magnetic atoms (four) in the volume $\mathrm{V}$ obtained by XRD, giving a magnetic spin density of $1.7391 \mathrm{e} 28 \mathrm{spins} / \mathrm{m}^{3}$. These values were 185 used to calculate the theoretical saturation magnetization. One can see that the experimental and theoretical values are in good agreement; the measured magnetisation value, $3.57 \mu_{\mathrm{B}}$ per unit formula, is around $97 \%$ of the theoretical saturation 190 magnetisation value, $3.67 \mu_{B}$ per unit formula.

In order to evaluate the specific heat under heating and cooling conditions, it was measured for different temperature rates, Fig. 3. The peak val-

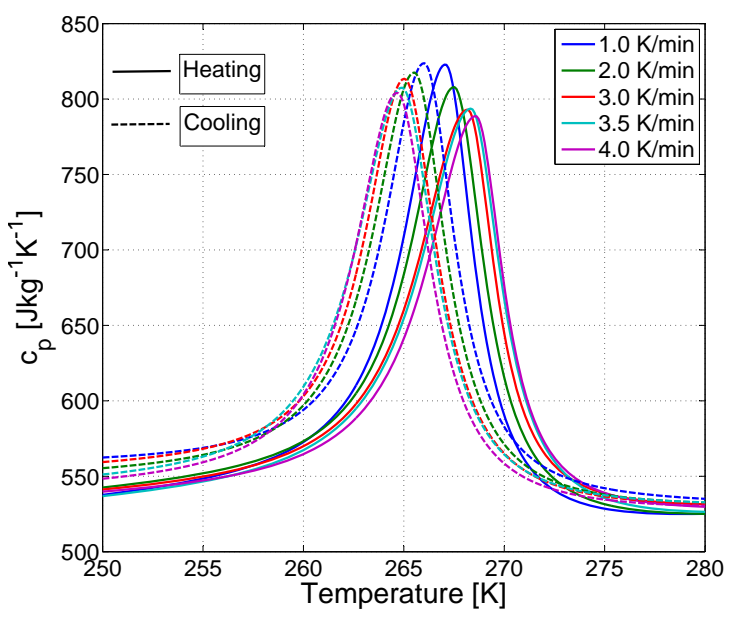

Figure 3: Specific heat as a function of temperature for different temperature rates at $0 \mathrm{~T}$.

ues and positions are in agreement with the literature [1, 5. The peak temperatures $\left(T_{\text {peak }}\right)$ of the $c_{p}$ curves were fitted as a function of rate in order to exclude the extrinsic hysteresis, as shown in Fig. 4. The observed hysteresis decreases with decreasing temperature rates, and for the extrapolated $0 \mathrm{~K} / \mathrm{min}$ rate, the intrinsic hysteresis is 0.08 $\mathrm{K}$, virtually zero considering the fitting and extrapolation uncertainties, $\pm 0.2 \mathrm{~K}$. It is interesting to notice that the expected $\Delta T_{\text {hyst }}$ for a rate of $5 \mathrm{~K} / \mathrm{min}$, is the same as the one reported elsewhere 11.

The extrinsic hysteresis under different temperature rates is related to the thermal diffusivity of the material. $\mathrm{La}_{0.33} \mathrm{Ca}_{0.67} \mathrm{MnO}_{3}$ has a large specific heat and a relatively low thermal conductivity, $1-3 \mathrm{Wm}^{-1} \mathrm{~K}^{-1}$ 20], which then leads to a low thermal diffusivity. Considering the base level of $c_{p}=$ $550 \mathrm{Jkg}^{-1} \mathrm{~K}^{-1}$, the density obtained from the XRD measurement $\rho=6034 \mathrm{kgm}^{-3}$ and assuming a value of $\kappa=2 \mathrm{Wm}^{-1} \mathrm{~K}^{-1}$, the thermal diffusivity can be calculated by $D=\kappa / c_{p} \rho$ and for $\mathrm{La}_{0.33} \mathrm{Ca}_{0.67} \mathrm{MnO}_{3}$ it is $6 \cdot 10^{-7} \mathrm{~m}^{2} \mathrm{~s}^{-1}$. This is approximately five times smaller than other common magnetocaloric materials such as $\mathrm{La}\left(\mathrm{Fe}_{0.88} \mathrm{Si}_{0.12}\right)_{13} \mathrm{H}_{1.0}$ where it is $\sim 2.7 \times 10^{-6} \mathrm{~m}^{2} \mathrm{~s}^{-1}$, very similar to the diffusivity of Gd 21. Therefore, the temperature rate is much more influential on the position of the peaks of $\mathrm{La}_{0.33} \mathrm{Ca}_{0.67} \mathrm{MnO}_{3}$ compared to other magnetocaloric materials.

To further investigate the thermal hysteresis, specific heat measurements under different applied fields were performed. Figure 5 shows the heat ca- 


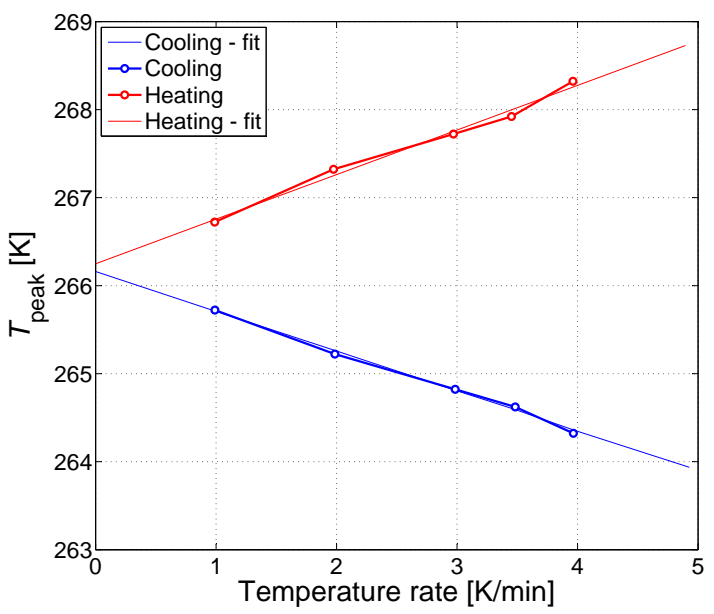

Figure 4: Heat capacity peak temperature, $T_{\text {peak }}$, as a function of temperature rates for both heating and cooling. At finite heating and cooling rates extrensic hysteresis is apparent. However, the intrinsic hysteresis, corresponding to the extrapolated temperature rate $0 \mathrm{~K} / \mathrm{min}$, vanishes.

pacity during the cooling procedure with $\dot{T}=-1.0$ $\mathrm{K} / \mathrm{min}$, under different applied fields. As the graph shows, the heat capacity peak is shifting towards higher temperatures for increasing values of $H$. This indicates a behaviour similar to that of a first order phase transition [22]. Figure 6 shows $T_{\text {peak }}$ as a function of field, for both heating and cooling procedures, with $|\dot{T}|=1.0 \mathrm{~K} / \mathrm{min}$. The linear fits show a slope of $6.2 \pm 0.9 \mathrm{~K} / \mathrm{T}$ for both the heating and cooling procedures. Furthermore, when extrapolating to a rate of $0 \mathrm{~K} / \mathrm{min}$ as in Figure 4 , the lines collapse showing the absence of hysteresis. In contrast, for materials with intrinsic hysteresis the amount of hysteresis decreases linearly with field, until a critical field is reached at which the transition becomes continuous and there is no more hysteresis.

Figure 7 shows indirect measurements of $\Delta s$ as a function of temperature for different values of $\Delta H$. One may see the asymmetric behaviour of the peak with increase in field; a behaviour related to first order transitions 22 . A slight hysteretic be- 24 haviour around the $T_{\text {peak }}$ is observed, with a $\Delta T_{\text {hyst }}$ of approximately $0.7 \mathrm{~K}$ in the full-width half maximum (FWHM). On the other hand, direct measurements of $\Delta s$ (see Figure 8) show no observable difference between the peak positions and, therefore, no significant thermal hysteresis was observed. The peak value is in agreement with literature values [5. The difference between the two types of measurements arises from the uncertainties related

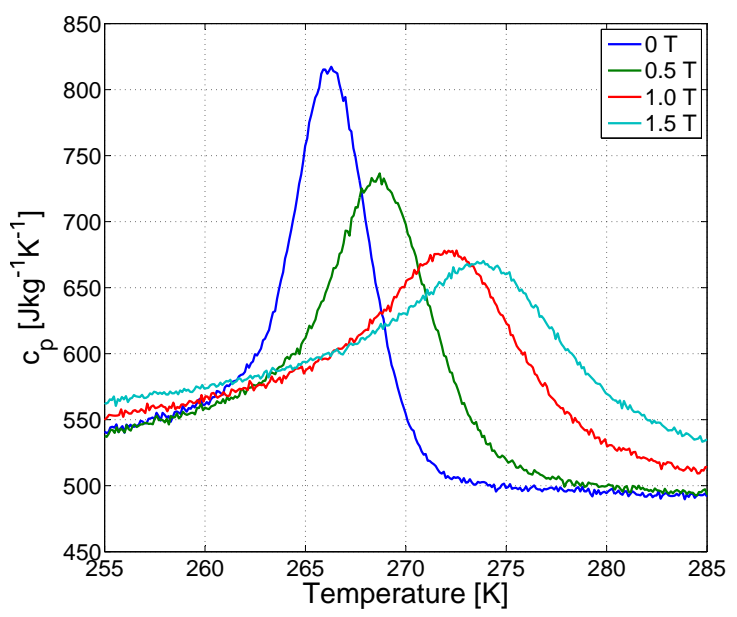

Figure 5: Specific heat as a function of temperature and under different applied field, with $\dot{T}=-1.0 \mathrm{~K} / \mathrm{min}$.

to the measurements. As Pecharsky and Gschneidner 23 have shown, $\Delta s$ derived from magnetisation measurements may have an uncertainty of up to $20 \%$, making it challenging to try to extract reliable values of a small or non-existent hysteresis. It is important to note that the direct measurement of $\Delta s$, is done with a high precision instrument with a temperature uncertainty of $\pm 10 \mathrm{mK}[16$. Furthermore, each point is measured individually, avoiding the smoothing effect observed when finite difference approximation is used to calculate $\Delta s$ from magnetisation derivatives.

\section{Discussion}

We find no discernible apparent hysteresis in LCMO, as shown above through the measurements 240 capacity and calorimetric measurements of entropy change. This is consistent with previous reports using an $\mathrm{AC}$ calorimetric method [5]. On the other hand the observed behaviour of the specific heat and isothermal entropy change is characteristic of a FOPT. These observations can be reconciled if we consider the spread in critical temperature which is caused by compositional variations and the tendency to formation of magnetically inhomogeneous states in the manganites [13. For weakly first-order materials even a small spread in critical temperatures may be enough to smooth out the transition and make the hysteresis disappear. Recently we showed, using detailed determination of the field dependence of $\Delta s$ at $T_{c}$ in combina- 


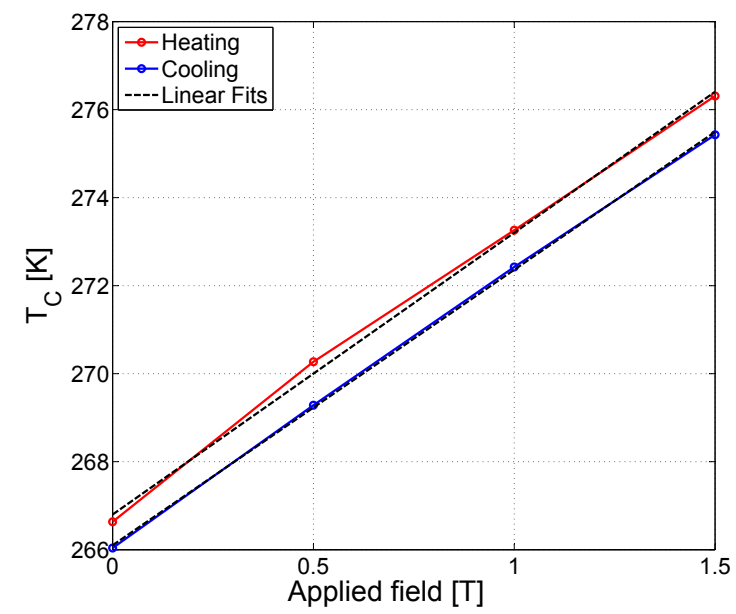

Figure 6: The specific heat peak temperature, $T_{\text {peak }}$, as a function of applied magnetic fields for both heating and cooling heat capacity measurements, with $|\dot{T}|=1.0 \mathrm{~K} / \mathrm{min}$. The dashed lines are linear fits.

tion with a fit to the Bean-Rodbell model that it is possible to determine the order of the phase transition and the spread in critical temperature [24]. We find indeed that $\mathrm{La}_{0.67} \mathrm{Ca}_{0.33} \mathrm{MnO}_{3}$ is weakly first order, with an $\eta=1.25$. In Figure 9 we show the modelled behaviour of the entropy curve under both heating and cooling, with a standard deviation on the Gaussian distribution $\sigma\left(T_{0}\right)=2.6 \mathrm{~K}$, and without any distribution. It is clear that the weak hysteresis present when there is no compositional inhomogeneity, i.e. $\sigma\left(T_{0}\right)=0 \mathrm{~K}$, tends to vanish when the spread is taken into consideration, i.e. $\sigma\left(T_{0}\right)=2.6 \mathrm{~K}$. It is also important to notice that the entropy curve shown here is in zero field. Previous works [22, 25, have shown that thermal hysteresis decreases with the increase of magnetic field, making it the largest at zero field. Moreover, the inset shows the entropy change under heating and cooling procedures, for both distributions and a magnetic field change of $1 \mathrm{~T}$. One may the great impact that the spread causes in the entropy change, making the peak wider and smaller. We have seen the same behaviour recently 26 in $\mathrm{La}(\mathrm{Fe}, \mathrm{Mn}, \mathrm{Si}){ }_{13} \mathrm{H}_{y}$. We believe such behaviour is due to the chemical inhomogeneity. If a material with mass $m$ has a single $T_{\mathrm{C}}$, all its mass will undergo the transition at that temperature, concentrating all the entropy change at it. However, if the material has a distribution in $T_{\mathrm{C}}$ due to, e.g., chemical inhomogeneity, different parts of the material will change from FM to PM at different temper-

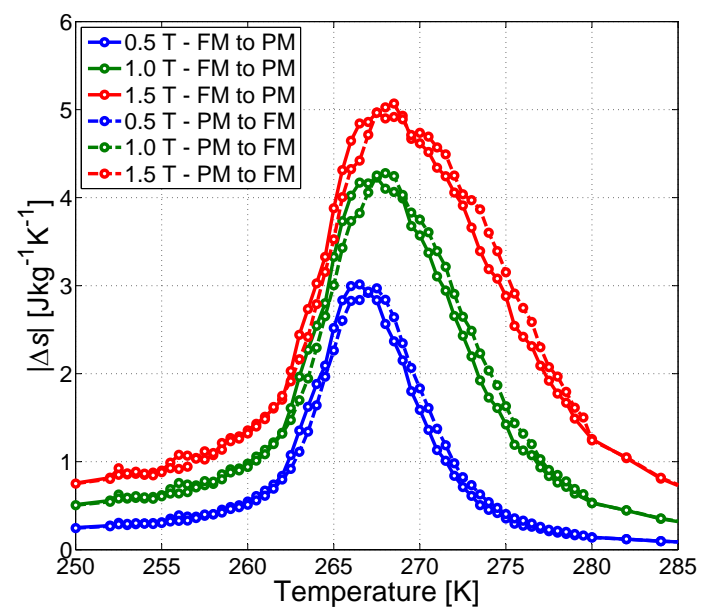

Figure 7: Entropy change calculated from magnetisation measurements from the VSM, using method (i) and (iii), from $0 \mathrm{~T}$ to $0.5,1.0$ and $1.5 \mathrm{~T}$.

atures, so the entropy change will be distributed accordingly to the distribution of $T_{\mathrm{C}}$.

\section{Conclusions}

We have studied the thermal hysteretic behaviour of $\mathrm{La}_{0.67} \mathrm{Ca}_{0.33} \mathrm{MnO}_{3}$ by means of several measurements and modelling. Direct $\Delta s$ measurements with $0.25 \mathrm{~K}$ step, measured in 4 different setups have shown no temperature difference. Heat capacity measured under $0 \mathrm{~T}$ and different temperature rates have shown no hysteresis when done the extrapolation to equilibrium state. Observed hysteresis does not decreases with field, pointing to the absence of intrinsic hysteresis. Still, FOPT behaviour was observed as $c_{p}$ shifting with field and the asymmetric growth of $\Delta s$ with increasing field changes. FOPT modelled with Bean-Rodbel model and with a superimposed Gaussian distribution on the $T_{\mathrm{C}}$ have shown that the spread in $T_{\mathrm{C}}$ can decrease signficantly, or even vanish the hysteresis.

\section{Acknowledgements}

This work was financed by the ENOVHEAT project which is funded by Innovation Fund Denmark (contract no 12-132673).

\section{References}

[1] G. Lin, Q. Wei, J. Zhang, J. Magn. Magn. Mater. 300 (2) (2006) 392-396. 


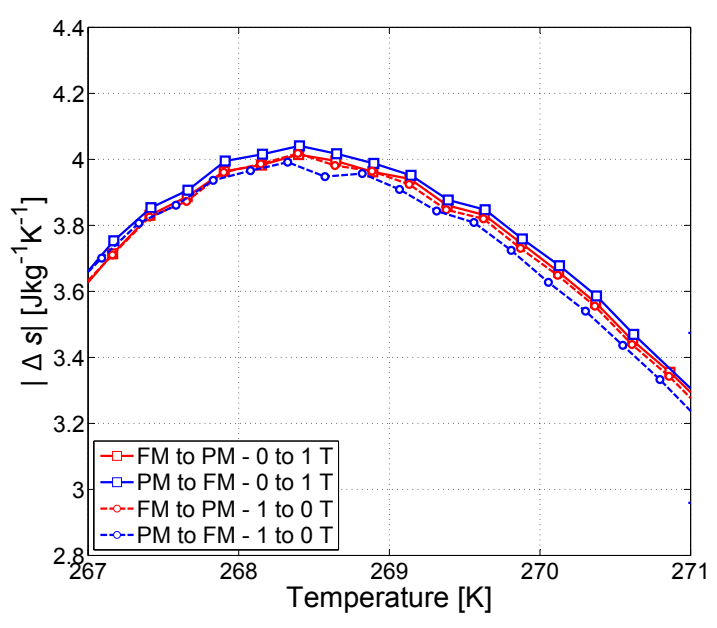

Figure 8: Entropy change measured directly in the DSC, around the transition temperature with a temperature step of $0.25 \mathrm{~K}$.

[2] Z. B. Guo, Y. W. Du, J. S. Zhu, H. Huang, W. P. Ding, D. Feng, Phys. Rev. B 78 (6) (1997) 1142-1145.

[3] H. Huang, Z. B. Guo, D. H. Wang, Y. W. Du, J. Magn. Magn. Mater. 173 (1997) 302-304.

[4] J. A. Turcaud, A. M. Pereira, K. G. Sandeman, J. S. Amaral, K. Morrison, A. Berenov, A. Daoud-Aladine, 355 L. F. Cohen, Phys. Rev. B 90 (2) (2014) 024410.

[5] K. Morrison, A. Berenov, L. F. Cohen, in: Mater. Res. Soc. Proc., Vol. 1310, 2011.

[6] L. Ewe, I. Hamadneh, H. a. Salama, R. Abd-Shukor, , Physica B 403 (13-16) (2008) 2394-2398.

[7] C. L. Canedy, K. B. Ibsen, G. Xiao, J. Z. Sun, a. Gupta, W. J. Gallagher, J. Appl. Phys. 79 (8) (1996) 4546.

[8] M.-H. Phan, S.-C. Yu, , J. Magn. Magn. Mater. 308 (2) (2007) 325-340.

[9] L. Caron, Z. Ou, T. Nguyen, D. Cam Thanh, O. Tegus, 365 E. Brück, J. Magn. Magn. Mater. 321 (21) (2009) 35593566.

330 [10] L. von Moos, C. R. H. Bahl, K. K. Nielsen, K. Engelbrecht, J. Phys. D: Appl. Phys. 48 (2015) 025005.

[11] C. P. Bean, D. S. Rodbell, Phys. Rev. 126 (1) (1962) 104-115.

[12] J. A. Turcaud, A. M. Pereira, L. F. Cohen, Phys. Rev. B 91 (13) (2015) 134410.

[13] N. G. Bebenin, R. I. Zainullina, V. V. Ustinov, J. Appl. Phys. 113 (2013) 073907.

[14] M. Kuepferling, C. P. Sasso, V. Basso, L. Giudici, IEEE Trans. Magn. 43 (6) (2007) 2764-2766.

[15] V. Basso, C. P. Sasso, M. Küpferling, Rev. Sci. Instrum. 81 (11) (2010) 113904.

[16] K. K. Nielsen, H. N. Bez, L. von Moos, R. Bjørk, D. Eriksen, C. R. H. Bahl, Rev. Sci. Instrum. 86 (10) (2015) 103903.

[17] S. Jeppesen, S. Linderoth, N. Pryds, L. T. Kuhn, J. B. Jensen, Rev. Sci. Instrum. 79 (8) (2008) 083901.

[18] A. Aharoni, J. Appl. Phys. 83 (6) (1998) 3432-3434.

[19] J. M. D. Coey, M. Viret, S. von Molnár, Adv. Phys. 48 (1999) 167-293.

350

[20] J. Turcaud, K. Morrison, a. Berenov, N. Alford, K. Sandeman, L. Cohen, Scr. Mater. 68 (7) (2013) 510-

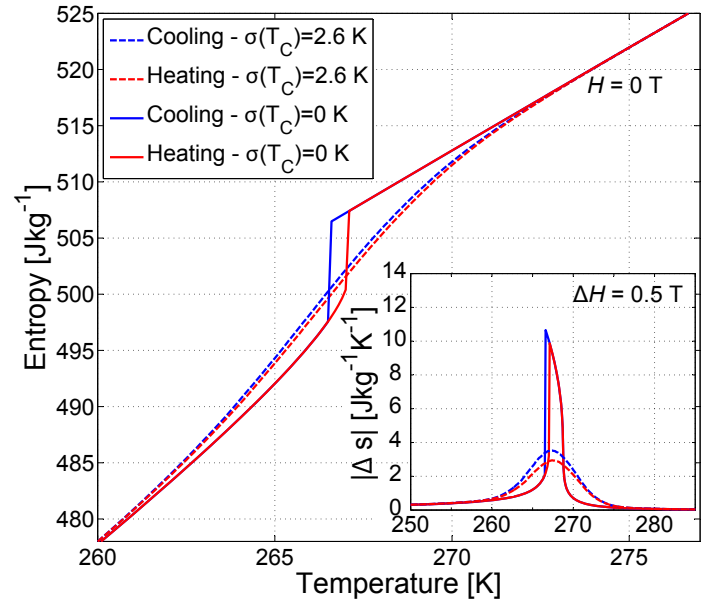

Figure 9: Entropy as function of the temperature, for heating and cooling procedures, and $H=0 \mathrm{~T}$. One may see the vanishing impact of the distribution $\sigma\left(T_{\mathrm{C}}\right)$ on the hysteresis. The inset shows the modelled isothermal entropy change for $\Delta H=0.5 \mathrm{~T}$, during heating and cooling procedures.

513.

[21] S. Fujieda, J. Appl. Phys. 95 (5) (2004) 2429

[22] A. Smith, C. R. H. Bahl, R. Bjørk, K. Engelbrecht, K. K. Nielsen, N. Pryds, Adv.Energy Mater. 2 (2012) 1288-1318.

[23] V. K. Pecharsky, K. a. Gschneidner, J.Appl. Phys. 86 (1) (1999) 565.

[24] A. Smith, K. K. Nielsen, H. N. Bez, C. R. H. Bahl, submitted to Phys. Rev. B (2015).

[25] V. Basso, M. Küpferling, C. Curcio, C. Bennati, A. Barzca, M. Katter, M. Bratko, E. Lovell, J. Turcaud, L. F. Cohen, Journal of Applied Physics 118 (5) (2015) 053907.

[26] H. N. Bez, K. K. Nielsen, P. Norby, A. Smith, C. R. H. Bahl, accepted in AIP Advances (2016). 